\title{
Sestrin2 integrates Akt and mTOR signaling to protect cells against energetic stress-induced death
}

\author{
I Ben-Sahra ${ }^{1,2,5}$, B Dirat ${ }^{1,2}, K_{\text {Laurent }}{ }^{1,2}$, A Puissant $^{3,6}$, P Auberger $^{2,3}$, A Budanov $^{4}$, J-F Tanti $^{1,2}$ and F Bost ${ }^{\star 1,2}$
}

The phosphoinositide-3 kinase/Akt (PI3K/Akt) pathway has a central role in cancer cell metabolism and proliferation. More importantly, it is one of the cardinal pro-survival pathways mediating resistance to apoptosis. The role of Akt in response to an energetic stress is presently unclear. Here, we show that Sestrin2 (Sesn2), also known as Hi95, a p53 target gene that protects cells against oxidative and genotoxic stresses, participates in the protective role of Akt in response to an energetic stress induced by 2-deoxyglucose (2-DG). Sesn2 is upregulated in response to an energetic stress such as 2-DG and metformin, and mediates the inhibition of mammalian target of rapamycin (mTOR), the major cellular regulator of energy metabolism. The increase of Sesn2 is independent of p53 but requires the anti-apoptotic pathway, PI3K/Akt. Inhibition of Akt, as well as loss of Sesn2, sensitizes cells to 2-DG-induced apoptosis. In addition, the rescue of Sesn2 partially reverses the pro-apoptotic effects of 2-DG. In conclusion, we identify Sesn2 as a new energetic stress sensor, which appears to be protective against energetic stressinduced apoptosis that integrates the pro-survival function of Akt and the negative regulation of mTOR.

Cell Death and Differentiation (2013) 20, 611-619; doi:10.1038/cdd.2012.157; published online 14 December 2012

Glucose is a major source of energy for cancer cells. Warburg demonstrated that cancer cells use preferentially glycolysis to generate ATP and have defective oxidative phosphorylation. ${ }^{1}$ A direct consequence of this discovery is the development of therapeutic strategies that specifically target cancer cell metabolism. 2-Deoxyglucose (2-DG) is the best known inhibitor of the glycolytic pathway. It is converted to phosphorylated 2-DG (2-DG-P) by the hexokinase, the first enzyme of glycolysis. 2-DG-P cannot be metabolized further in the glycolysis pathway and thus accumulates intracellularly and inhibits the hexokinase or can alternatively use the pentose phosphate pathway. The inhibition of glycolysis leads to ATP depletion and energetic stress. ${ }^{2}$ Cells require nutrients for their energetic needs and to support the biosynthetic pathway necessary for their growth and proliferation. This led to the hypothesis that glucose deprivation sensitizes cancer cells to apoptosis. ${ }^{3}$ Indeed, several studies have shown that 2 DG induces apoptosis in cancer cells. ${ }^{4-8}$

Energetic stress activates the AMP-activated protein kinase (AMPK), a stress sensor protein kinase, which downregulates the mammalian target of rapamycin (mTOR). mTOR is a master switch of anabolism and catabolism. ${ }^{9}$ Indeed, to avoid death and energy shortage, cells slow down their metabolism and inhibit anabolic reactions via the downregulation of the mTOR pathway. Other biological processes implicated in the resistance to apoptosis involve the phosphoinositide-3 kinase/Akt (PI3K/Akt) pathway. The $\mathrm{PI}$ KK/Akt is a major pro-survival pathway. Its constitutive activation by growth factors, mutation of PI3K and inactivation of PTEN (phosphatase and tensin homolog) promotes cell growth and is a characteristic of numerous cancer cells. ${ }^{10}$ One of the first identified Akt target with direct implications for regulating cell survival is the pro-apoptotic $\mathrm{Bcl}-2$ family member BAD. Once phosphorylated, the phosphoserine residues of $B A D$ form affinity-binding sites for 14-3-3 molecules, thus localizing phosphorylated Bad to the cytosol and effectively neutralizing its pro-apoptotic activity. ${ }^{11}$ Other targets such as cytochrome $\mathrm{C}$ and FOXO1a have been described as mediators of the Akt anti-apoptotic response. $^{12,13}$ While AMPK inhibits mTOR, Akt positively regulates $m$ TOR via the phosphorylation and the inhibition of tuberous sclerosis protein complex 2 (TSC2), and promotes anabolic reactions required for cell growth and proliferation. ${ }^{14}$

Sestrin2 (Sesn2) negatively regulates mTOR signaling and executes this function through the activation of AMPK and TSC2 phosphorylation. ${ }^{15}$ Sesn2 is implicated in the cellular response to oxidative stress and DNA damage. It exerts its cytoprotective function by regenerating peroxiredoxins, having therefore a major role in the antioxidant defense of the cell. Sesn2 is a target of the tumor suppressor gene p53 and was

\footnotetext{
${ }^{1}$ INSERM U1065, Centre Méditerranéen de Médecine Moléculaire (C3M), Team Cellular and Molecular Physiopathology of Obesity and Diabetes, Nice, France; ${ }^{2}$ Université de Nice Sophia-Antipolis, Faculté de Médecine, Nice, France; ${ }^{3}$ INSERM U1065, Centre Méditerranéen de Médecine Moléculaire (C3M), Team Cell Death Differentiation and Cancer, Nice, France and ${ }^{4}$ Massey Cancer Center, Department of Neurosurgery, Virginia Commonwealth University, Richmond, VA, USA ${ }^{*}$ Corresponding author: F Bost, INSERM, U1065, Centre Mediterraneen de Medecine Moleculaire (C3M), Team Cellular and Molecular Physiopathology of Obesity and Diabetes, 151 route de St Antoine de Ginestière, Nice F-06204, France. Tel: +33 4890 64229; Fax: +33 4890 64221; E-mail: bost@ unice.fr

${ }^{5}$ Current address: Harvard School of Public Health, Department of Genetics and Complex diseases, Boston, MA 02115, USA.

${ }^{6}$ Current address: Dana-Farber Cancer Institute, Pediatric-Oncology Department, Boston, MA 02215, USA.

Keywords: cell metabolism; AMPK; apoptosis; 2-deoxyglucose; metformin; sestrin

Abbreviations: 2-DG, 2-deoxyglucose; AICAR, 5-amino-1- $\beta$-D-ribofuranosyl-imidazole-4-carboxamide; AMPK, AMP-activated protein kinase; ER, endoplasmic reticulum; GSK3, glycogen synthase 3; HBSS, Hanks buffered salt solution; mTOR, mammalian target of rapamycin; mTORC1, mTOR complex 1; PARP, poly (ADPribose) polymerase; PI3K, phosphoinositide-3 kinase; PTEN, phosphatase and tensin homolog; ROS, reactive oxygen species; TSC2, tuberous sclerosis protein 2 Received 23.4.12; revised 22.10.12; accepted 8.11.12; Edited by N Chandel; published online 14.12 .12
} 
shown to provide an important link between genotoxic stress, p53 and the mTOR pathway. ${ }^{15}$ However, the role of Sesn2 in the cellular response to an energetic stress is presently unknown. Given the important role of Sesn2 in response to genotoxic and oxidative stresses, and its role in the regulation of the AMPK/mTOR axis, we chose to determine the role of Sesn2 in energetic stress-induced apoptosis. We show that 2DG induced ATP depletion and upregulated Sesn2 expression in an Akt-dependent but p53-independent way. We also provide evidence that Sesn2 is required for mTOR inhibition in response to an energetic stress. Finally, we demonstrate that Sesn2 is a new mediator of energetic stress, which protects against 2-DG-induced apoptosis and participates to the protective role of Akt.

\section{Results}

2-DG induces energetic stress and apoptosis. LNCaP cells were treated with high concentration of the inhibitor of glycolysis 2-DG $(20 \mathrm{mM})$ or incubated in the Hank's buffered salt solution (HBSS) for $8 \mathrm{~h}$ to induce an energetic stress. Both treatments led to a strong decrease in the intracellular ATP concentration (Figure 1a). We then determined if this energetic stress triggered apoptosis. 2-DG stimulated caspase 3 activity and induced the cleavage of poly (ADP-ribose) polymerase (PARP) and pro-Caspase 3, two markers of apoptosis, in LNCaP (Figures $1 \mathrm{~b}$ and $\mathrm{c}$ ), DU145, PC3 and A549 cells (Supplementary Figures S1A and B). To determine the implication of caspases in 2-DG-induced apoptosis, LNCaP cells were treated with the pan-caspase inhibitor Z-VAD-fmk. Such a treatment drastically inhibited the cleavage of PARP and pro-caspase 3 induced by 2-DG (Figure 1c). These results demonstrate that the treatment with 2-DG induced an energetic stress and is associated with the induction of caspase-dependent cell death.

2-DG induces the activation of Akt. Akt is a pro-survival and anti-apoptotic pathway, ${ }^{16}$ and 2-DG has been shown to phosphorylate Akt in cancer cells. ${ }^{17}$ The full activation of Akt requires its phosphorylation on two sites, Ser ${ }^{473}$ and $\mathrm{Thr}^{308}$. We show that 2-DG stimulates the phosphorylation on both sites from 4 to $24 \mathrm{~h}$ (Figure 2a). The Akt activation was confirmed as 2-DG induced the phosphorylation of its substrates, the glycogen synthase kinase 3 (glycogen synthase 3 (GSK3)) and TSC2, on Thr 1462 (Figure 2b). In order to determine if Akt exerts a protective effect against 2DG-induced apoptosis, we invalidated Akt1 and Akt2 with siRNA against the two isoforms. The inhibition of Akt led to an increase of caspase 3 activity upon 2-DG treatment demonstrating that Akt protects against 2-DG-induced apoptosis (Figure 2c and Supplementary Figure S2).

Sesn2 is induced by 2-DG and is important for energeticstress-induced inhibition of mTOR. Sesn2 is a stressinduced protein that regulates the AMPK/mTOR axis, a major sensor of the energetic level of the cell. To determine if Sesn2 is modulated by an energetic stress, cells were treated with 2-DG and HBSS. Both conditions increased Sesn2 expression, and as expected, this energetic stress led a
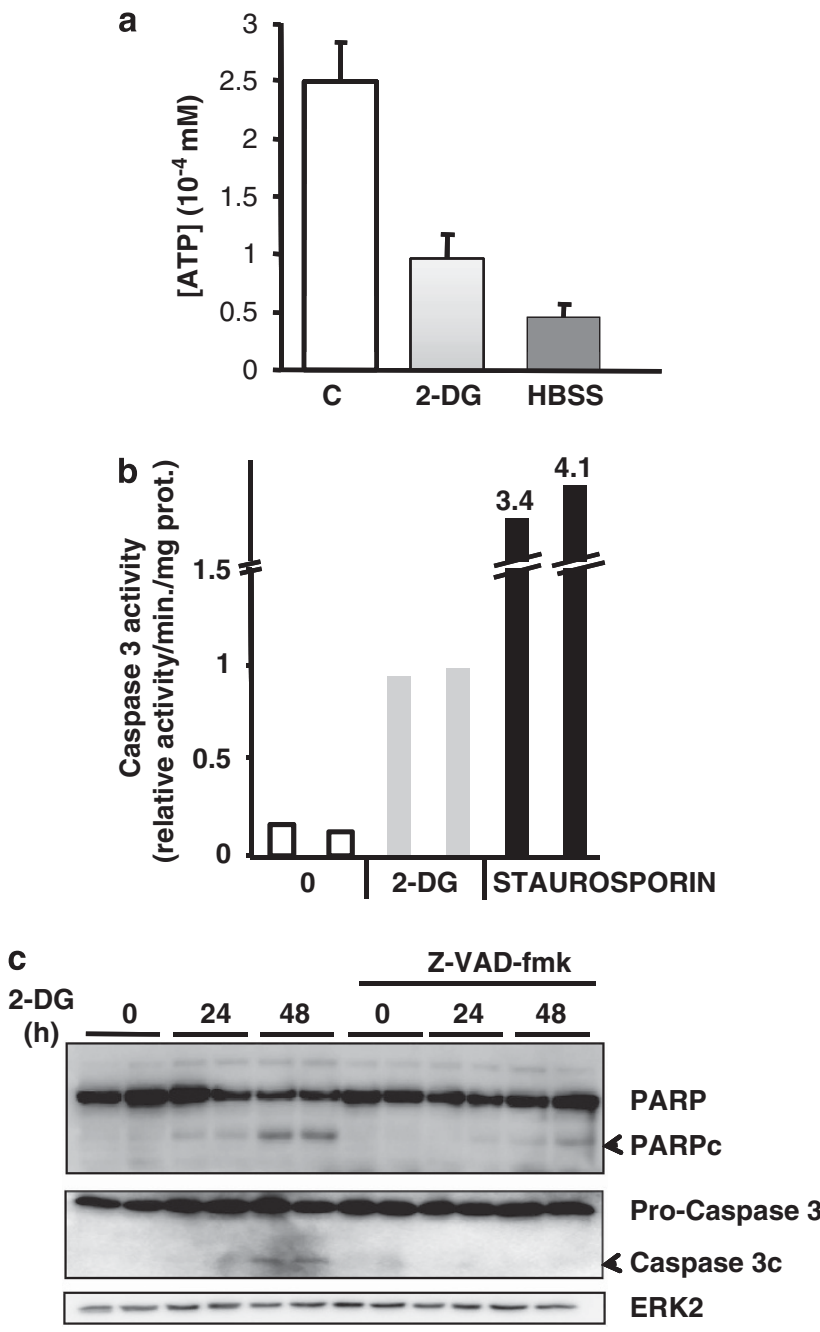

Figure 1 2-DG induces caspase-depenent apoptosis in LNCaP. (a) Intracellular ATP concentration in LNCaP cells treated with $20 \mathrm{mM} 2-\mathrm{DG}$ or incubated in HBSS for $8 \mathrm{~h}$. (b) Measurement of caspase 3 activity in LNCaP cells treated with $20 \mathrm{mM}$ 2$D G$ during $48 \mathrm{~h}$ or staurosporin $(1 \mu \mathrm{M})$ for $24 \mathrm{~h}$. The figure is representative of one experiment performed three times with each condition in duplicate. (c) LNCaP cells were treated with $20 \mathrm{mM}$ 2-DG for 24 or $48 \mathrm{~h}$ in the presence or absence of ZVADfmk $(100 \mu \mathrm{M})$

to the inhibition of phosphorylation of S6 ribosomal protein, a marker of mTOR activation (Figure 3a). Of note, Sesn2 antibodies detect two bands, but only the high molecular weight band ( $56 \mathrm{kDa})$ corresponds to Sesn2, as illustrated in Figure 3c where Sesn2 siRNA decreases only the upper band. To determine if the cellular response to the energetic stress is reversible, cells were treated with 2-DG or incubated with HBSS for $15 \mathrm{~h}$ and then the media was changed to regular growth media for $24 \mathrm{~h}$. When the energetic stress is removed, Sesn2 protein levels, as well as the phosphorylation of S6 ribosomal protein, returned to the control level (Figure 3a). These results indicate that the energetic stress inducers reversibly control Sesn2 expression. Similarly to Sesn2, Sesn3 has been shown to inhibit mTOR complex 1 (mTORC1), and Akt affects Sesn3 expression through FoxO1. ${ }^{18}$ We analyzed Sesn3 expression in response to 

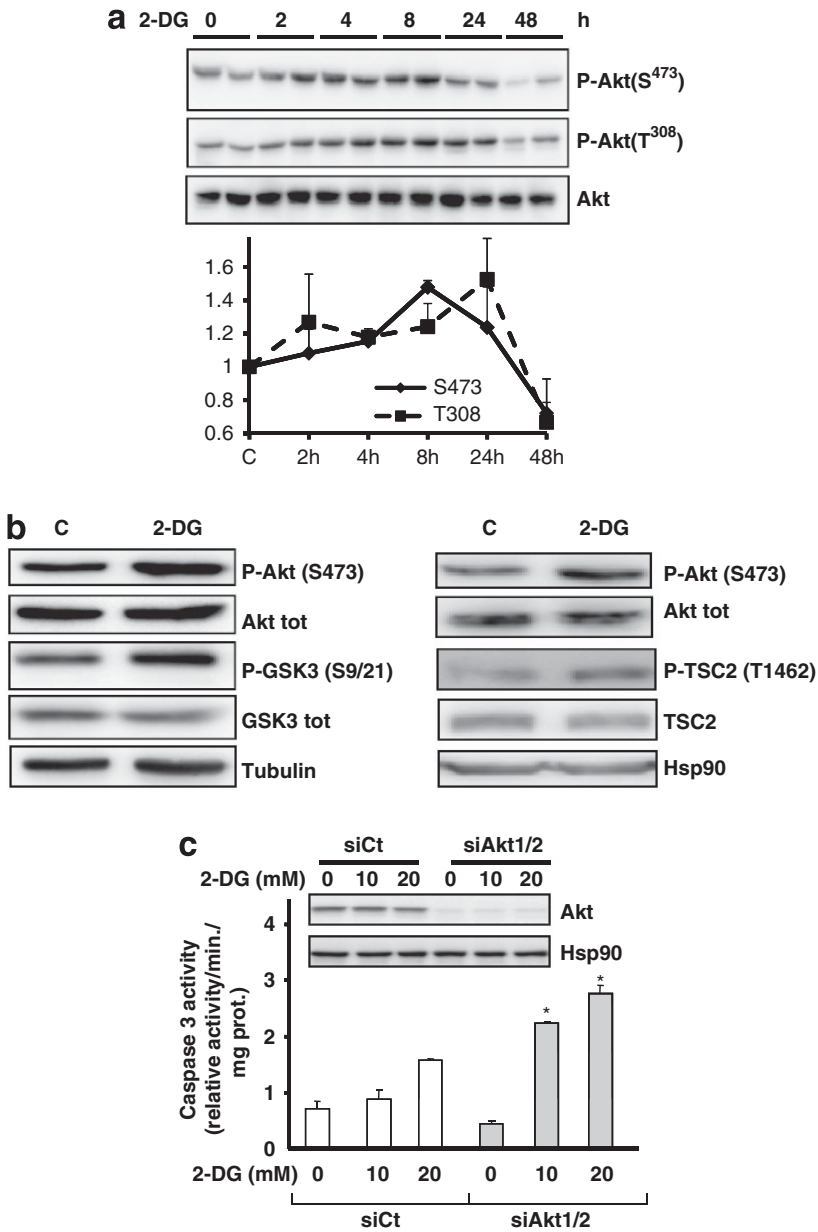

Figure 2 2-DG induces the activation of Akt. (a) LNCaP were incubated with $20 \mathrm{mM}$ 2-DG for the indicated times, and an immunoblot of PAkt Ser ${ }^{473}$ and $\mathrm{Thr}^{308}$ was performed. The graph represents the quantification of three independent experiments. (b) LNCaP were treated with $20 \mathrm{mM}$ 2-DG for $8 \mathrm{~h}$ before the immunobloting of the indicated proteins. (c) LNCaP cells were transfected with siRNA against Akt1 and Akt2 for $48 \mathrm{~h}$ and treated with 2-DG for $48 \mathrm{~h}$ before the caspase 3 assay. The immunoblot represents the level of Akt before the caspase assay. The experiment is representative of three independent experiments, with each condition in triplicate. The differences were significant with $P<0.05\left(^{*}\right)$

different stimuli, and we show that Sesn3 expression is not affected by 2-DG and the inhibitor of PI3K, LY294002. On contrary, we observed an increase of Sesn3 expression with HBSS and in the condition of serum starvation (Figure $3 b$ ). To determine the role of Sesn2 in mTOR inhibition in response to an energetic stress, we invalidated Sesn2 in LNCaP cells using siRNA. 2-DG and rapamycin (a direct and specific inhibitor of $\mathrm{mTORC} 1$ ) inhibited the phosphorylation of S6 ribosomal and 4EBP1 in cells transfected with siCt or in non-transfected cells (Supplementary Figure S3). Invalidation of Sesn2 reversed the inhibition of S6 phosphorylation and 4EBP1 induced by 2-DG (Figure $3 \mathrm{c}$ and Supplementary Figure S3A). The downregulation of Sesn2 had no effect on mTOR activity in basal conditions and did not affect the inhibition of mTOR by rapamycin. We then analyzed mTOR activity in Sesn2 $2^{-/}$mouse embryonic fibroblasts (MEFs) upon 2-DG and metformin treatment. Metformin is an anti-diabetic drug that decreases intracellular ATP concentration as described previously. ${ }^{19}$ Similarly to $2-\mathrm{DG}$, metformin increased Sesn2 expression. Both agents inhibited mTOR activity in wild-type (WT) MEFs but not in Sesn2 ${ }^{-1-}$ MEFs (Figure 3d and Supplementary Figure S3B). On contrary, the inhibition of phospho-S6 ribosomal protein was not reversed in Sesn $2-/$ - cells in response to HBSS. Altogether, these results demonstrate that Sesn2 is required for the inhibition of mTOR induced by an energetic stress induced by 2-DG and metformin but not by HBSS.

Sesn2 response to an energetic stress is independent of p53. p53 has been shown to upregulate Sesn2 expression in reponse to genotoxic and oxidative stresses. ${ }^{15,20}$ To determine if $p 53$ is implicated in Sesn2 increase in response to an energetic stress, we invalidated p53 in LNCaP cells. The downregulation of p53 did not alter 2-DG-induced Sesn2 expression, whereas as expected, it blocked its increase induced by bleomycin (a DNA damage inducer) (Figure 4a). We also used different cancer cells harboring WT p53 (LNCaP), mutated p53 (DU145) or invalidated for p53 (PC3). Whatever the status of p53, 2-DG increased the expression of Sesn2 and decreased the phosphorylation of S6 ribosomal protein in the three cell lines (Figure 4b). Similarly, 2-DG was able to induce Sesn2 expression in MEFs invalidated for p53 (Supplementary Figure S4A). To overrule the possibility that 2-DG indirectly regulates Sesn2 expression, we measured the formation of reactive oxygen species (ROS) in response to 2-DG and HBSS. 2-DG and HBSS did not alter ROS levels while as expected, $\mathrm{H}_{2} \mathrm{O}_{2}$ increased ROS formation and this was inhibited by $\mathrm{N}$-acetyl cysteine (Supplementary Figure $\mathrm{S} 4 \mathrm{~B})$. Altogether, these results demonstrate that the upregulation of Sesn2 is independent of p53 in response to an energetic stress.

Akt is required for energetic stress-induced Sesn2 expression. We have shown that Akt is phosphorylated and activated in response to 2-DG (Figures $2 a$ and b). To determine if the PI3K/Akt pathway modulates Sesn2 expression in response to an energetic stress, cells were treated with LY294002, an inhibitor of PI3K. LY294002 efficiently abolished the phosphorylation of Akt in LNCaP (Figure 4c). Interestingly, in the presence of LY294002 or an inhibitor of Akt1/2, 2-DG no longer increased Sesn2 expression in LNCaP, PC3 and DU145 (Figure 4c, Supplementary Figures S5A, B and C). We then used siRNA against Akt1 and Akt2. While Akt was efficiently knocked down, Sesn2 expression decreased in control conditions and did not increase in response to 2-DG (Figure $4 d$ ). In order to determine if the increase of Sesn2 is transcriptionally regulated, we analyzed Sesn2 mRNA levels in LNCaP cells, in which Akt1/2 were downregulated, and in cells treated with LY294002. 2-DG strongly increased Sesn2 mRNA levels, and inhibition of Akt did not significantly affect Sesn2 mRNA basal levels. Downregulation of Akt or inhibition of PI3K, partially but significantly, decreased the level of Sesn2 induced by 2-DG (Supplementary Figure S5D), suggesting that the increase of Sesn2 induced by $2-D G$ is regulated in part by Akt. Altogether, these results clearly demonstrate that the PI3K/ Akt pathway controls Sesn2 in response to 2-DG. 

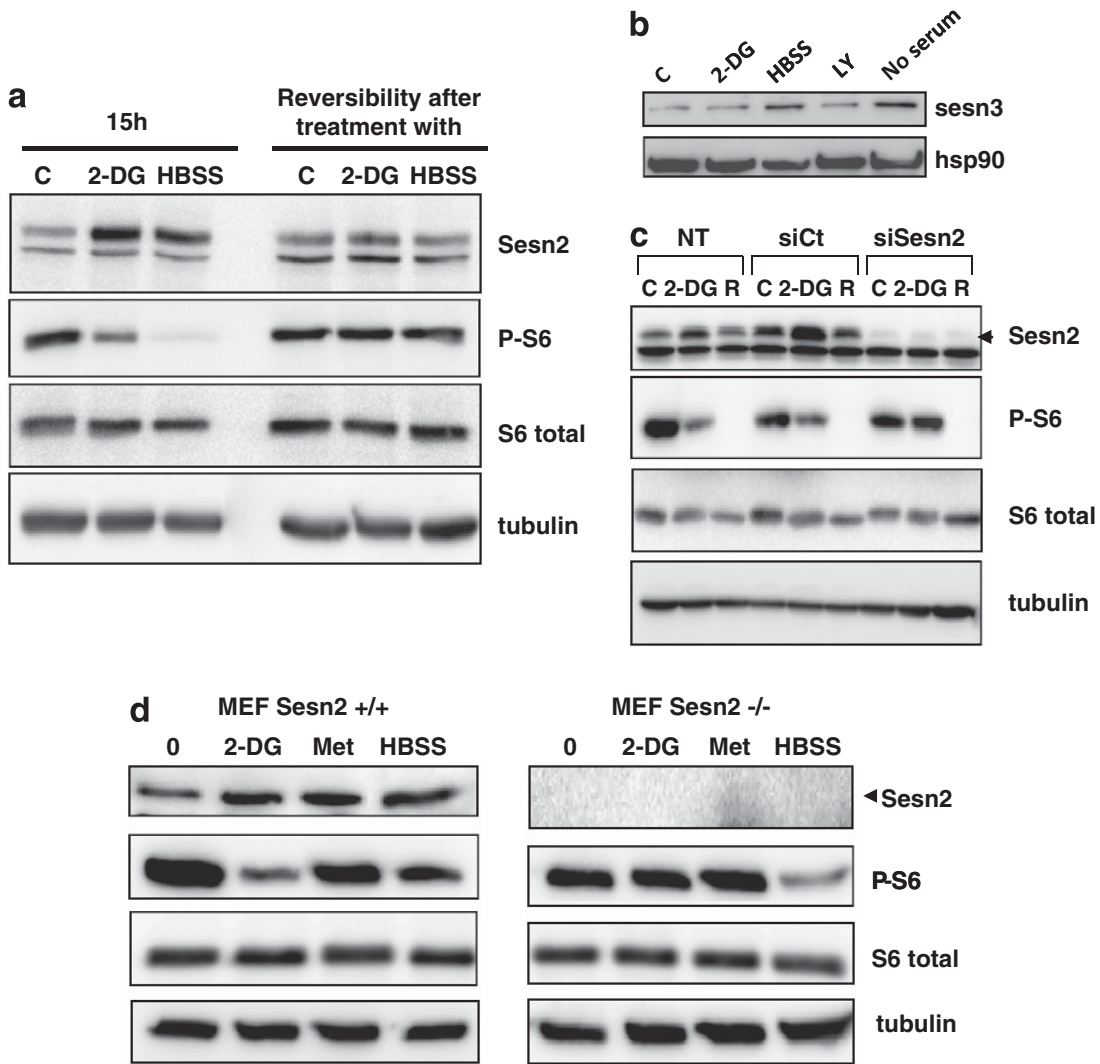

Figure 3 Sesn2 is required for the downregulation of mTOR by 2-DG. (a) LNCaP cells were untreated or treated with $20 \mathrm{mM}$ 2-DG or incubated with HBSS for $15 \mathrm{~h}$. To test the reversibity after the treatment, cells were cultivated in complete medium for $24 \mathrm{~h}$ before immunoblotting. (b) LNCaP cells were treated with $20 \mathrm{mM} 2-\mathrm{DG}, 50 \mu \mathrm{M} \mathrm{LY} 294002$ or incubated with HBSS or no serum for $24 \mathrm{~h}$ before immunoblotting. (c) LNCaP cells were transfected with Sesn2 or control siRNA (SiCt), or not (NT). At $48 \mathrm{~h}$ after the transfection, cells were treated with $20 \mathrm{mM} 2-\mathrm{DG}$ or $40 \mathrm{nM}$ rapamycin for $15 \mathrm{~h}(\mathrm{R})$. (d) Immunoblotting of WT $\left(\right.$ Sesn2 ${ }^{+/+}$) or Sesn2 ${ }^{-}{ }^{-}$MEFs treated with $20 \mathrm{mM} 2-\mathrm{DG}$, $5 \mathrm{mM}$ metformin (Met) or grown in HBSS for $15 \mathrm{~h}$

Sesn2 protects against 2-DG-induced apoptosis. From the above results, we hypothesized that Akt activation is a survival process against 2-DG-induced apoptosis and that Sesn2 is implicated in this protective response. To validate this hypothesis, we downregulated Sesn2 expression in LNCaP cells using three different siRNA targeting different regions of Sesn2. The three siRNA efficiently abolished Sesn2 expression, and invalidation of Sesn2 resulted in an increase of PARP cleavage in basal conditions and in response to 2-DG (Figure 5a). In parallel, the downregulation of Sesn2 significantly increased caspase 3 activity in control conditions and in response to 2-DG (Figure $5 b$ ). To further validate the implication of Sesn2 in the protection against apoptosis, we performed an annexin V/propidium iodide (PI) labeling assay, and we demonstrated that invalidation of Sesn2 sensitized cancer cells to 2-DG-induced apoptosis (Figure 5c). Endoplasmic reticulum (ER) stress response has been shown to mediate apoptosis induced by 2-DG in rhabdomyosarcoma cells. ${ }^{21}$ We demonstrate that tunicamycin (positive control for ER stress) and to a less extend 2-DG induced the expression of GRP78/Bip (a specific marker of ER stress) in LNCaP cells (Supplementary Figure S6A). Importantly, siRNA-mediated invalidation of Sesn2 did not affect the increase of GRP78 induced by 2-DG, while it augments GRP78 expression in response to tunicamycin. These results suggest that Sesn2 does not interfere with
2-DG-induced ER stress in LNCaP cells but may sensitize LNCaP cells to tunicamycin-induced ER stress (Supplementary Figure S6B). We then determined if MEFs invalidated for Sesn2 were more sensitive to 2-DG-induced apoptosis. Interestingly, we observed an increase of the cleaved forms of PARP and caspase 3 in Sesn2 ${ }^{-1-}$ MEFs compared with WT MEFs in response to 2-DG (Figure 5d, Supplementary Figure S6C) and Sesn2 ${ }^{-1-}$ cells displayed a higher caspase 3 activity than WT cells treated with 2-DG (Supplementary Figure S6D). In accordance with these results, 2-DG and HBSS decreased more significantly the viability of MEFs invalidated for Sesn2 compared with WT (Supplementary Figure S6E). Then, we restored Sesn2 expression in cells invalidated for Sesn2 and 2-DG no longer increased PARP and Caspase 3 cleavage in cells rescued for Sesn2 (Figure 5e). Altogether, our results demonstrate that invalidation of Sesn2 sensitizes cells to 2-DG induced apoptosis.

Sesn2 knockdown affects AMPK activity and intracellular ATP levels. Energetic stress activates AMPK and leads to the phosphorylation of the acetyl CoA carboxylase (ACC), a direct substrate of AMPK (Figure 6a). To determine whether Sesn2 regulates AMPK activity in response to 2-DG, we knocked down Sesn2 in LNCaP cells and analyzed the phosphorylation of ACC. As expected, 2-DG decreased the 

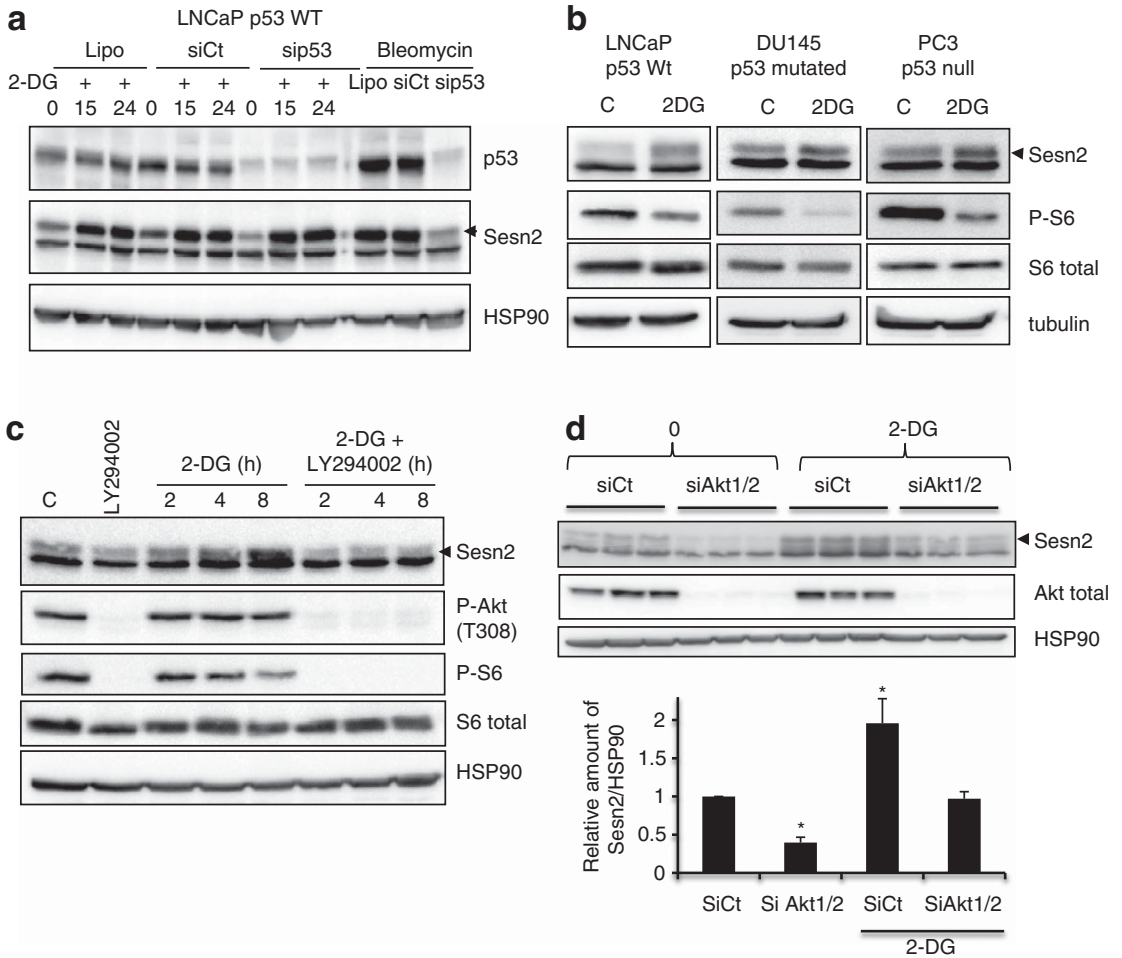

Figure 4 The Sesn2 response to an energetic stress is regulated by Akt. (a) LNCaP cells were either treated with lipofectamine (Lipo) or transfected with siCt or sip53 for $48 \mathrm{~h}$. Then, $20 \mathrm{mM}$ 2-DG was added for $15 \mathrm{~h}$ (2-DG15) or $24 \mathrm{~h} \mathrm{(2-DG24),} \mathrm{and} \mathrm{bleomycin} \mathrm{(10} \mu \mathrm{g} / \mathrm{ml})$ for $24 \mathrm{~h}$. Immunoblotting was performed using Sesn2, p53 and hsp90 antibodies. (b) Immunoblotting of Sesn2, P-S6 ribosomal protein, S6 ribosomal and tubulin in three prostate cancer cell lines with different p53 status. (c) Western blot analysis of LNCaP cells treated or not with the LY294002 $(50 \mu \mathrm{M})$ and with $20 \mathrm{mM}$ 2-DG for the indicated time. (d) LNCaP cells were transfected with siCt, or siAkt1 + siAkt2, after $48 \mathrm{~h}$, $20 \mathrm{mM}$ 2-DG was added to the cells for $24 \mathrm{~h}$. The figure represents the relative amount of Sesn2 and is the mean of three independent experiments. The differences were significant with ${ }^{*} P<0.05$

phosphorylation of S6 ribosomal and induced the phosphorylation of ACC on $\mathrm{Ser}^{79}$. Invalidation of Sesn2 reversed the inhibitory effect of 2-DG on the phosphorylation of S6 ribosomal (Figure 6a). Interestingly, the knockdown of Sesn2 led to a significant reduction of 2-DG-induced phosphorylation of ACC suggesting that, in response to an energetic stress, Sesn2 potentiates the activity of AMPK. AMPK is a sensor of the energetic status of the cell sensitive to intracellular ATP concentration. In order to determine if the variation of Sesn2 expression affects the energetic status of the cell, we measured ATP concentration in LNCaP cells transfected with Sesn2 siRNA. While 2-DG lead to a decrease of ATP after $8 \mathrm{~h}$ of treatment, the downregulation of Sesn2 led to a stronger and significant decrease of ATP concentration compared with cells transfected with siCt (Figure $6 \mathrm{~b}$ ). Interestingly, the knocking down of Sesn2 by itself decreases ATP concentration significantly $(P=0.003)$ (Figure 6b). Altogether, these results suggest that Sesn2 protects against ATP depletion.

Our results demonstrate that Sesn2 protects against 2-DGinduced apoptosis and participates to the pro-survival role of Akt by regulating mTOR signaling.

\section{Discussion}

As cancer cells largely depend on glycolysis for their energetic needs, inhibition of this metabolic pathway is an important issue in cancer therapy. ${ }^{22}$ Glucose deprivation causes decrease of ATP, and inhibition of glycolysis induces apoptosis in cancer cells. ${ }^{23}$ We confirm that 2-DG markedly decreased intracellular ATP concentration together with the induction of apoptosis in several cancer cell lines. Consecutively, the prosurvival kinase Akt is phosphorylated on Ser ${ }^{473}$ and $\mathrm{Thr}^{308}$ and activated. We demonstrate that inhibition of Akt sensitizes cancer cells to 2-DG-induced apoptosis. Typically, growth factors such as insulin and nerve growth factor activate the PI3K/Akt pathway to protect cells against apoptosis, and inhibition of this pathway triggers apoptosis. ${ }^{24}$ In this study, we demonstrate that the activation of Akt is an adaptative response and protective mechanism against glycolysis inhibition. The mechanism of Akt protection in response to cellular stress has been previously described. For example, Wei and Vander Heide ${ }^{25}$ showed that heat shock activates Akt to protect cardiomyocytes from heat stressinduced cell lethality; similarly, Akt is activated in $\mathrm{C} 2 \mathrm{C} 12$ cells subjected to mitochondrial stress generated by the depletion of mtDNA, and Akt1 silencing sensitizes cells to apoptosis. ${ }^{26}$ Akt regulates several proteins, such as Foxo3, GSK3 $\beta$, Chk1 and $\mathrm{Bad}$, which participate to its protective role against apoptosis. ${ }^{27}$ Indeed, the phosphorylation of these proteins inhibits their functions in inducing apoptosis, causing cell cycle arrest and establishing DNA damage checkpoint. ${ }^{27}$ 2-DG has been shown to activate Akt in several cancer cell lines (see Zhong et al. ${ }^{17}$ and Supplementary Figures S5B and C). Zhong 


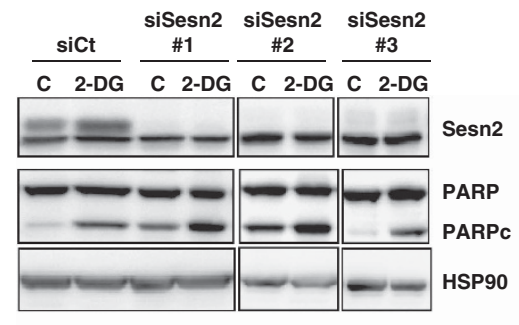

b

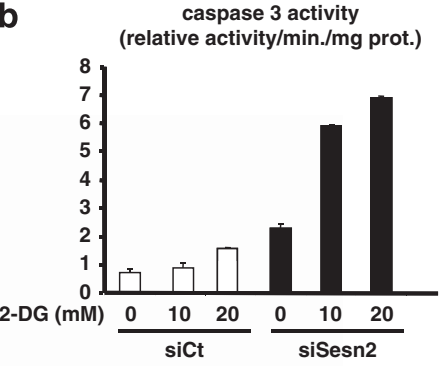

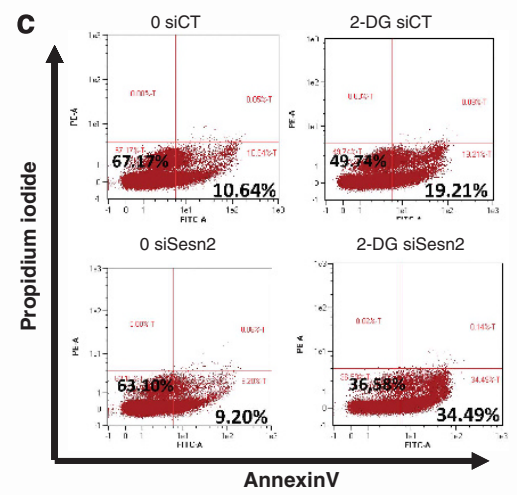

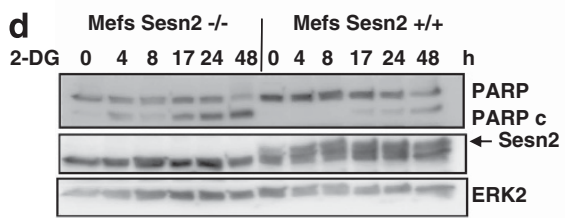

e Mefs Sesn2 -/PCMV Flag Sesn2

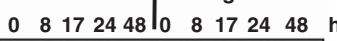
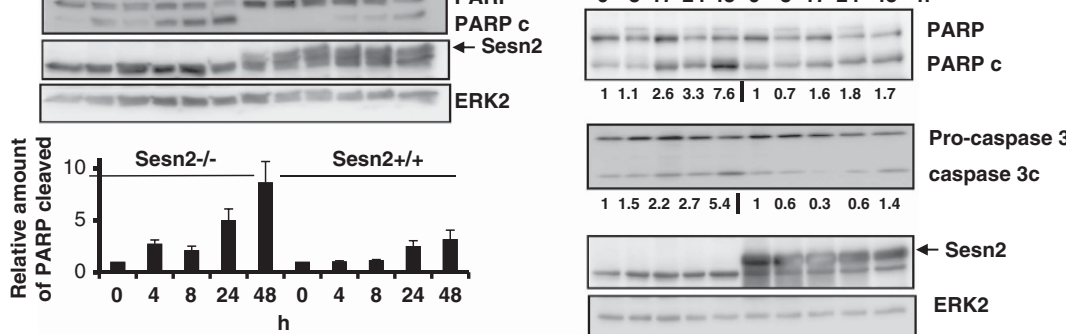

Figure 5 Sesn2 protects against 2-DG-induced apoptosis. (a) LNCaP cells were transfected with three different siRNA targeting Sesn2 for $48 \mathrm{~h}$ and a western blot analysis was performed after the treatment with 2-DG for $24 \mathrm{~h}$. (b) Measurement of caspase 3 activity in cells transfected with siCt or siSesn2 and treated with 2-DG $20 \mathrm{mM}$ for $48 \mathrm{~h}$. (c) Flow cytometry analysis of LNCaP cells transfected with siCt or siSesn2, treated with 2-DG $20 \mathrm{mM}$ for $24 \mathrm{~h}$ and labeled with Annexin V/PI. The percentage of cells positively labeled with Annexin $V$ is indicated for each condition. (d) WT or Sesn2 ${ }^{-1-}$ MEFs were treated with $20 \mathrm{mM}$ 2-DG for the indicated time; the graph represents the quantification of the amount of the cleaved form of PARP in three independent experiments (mean $+I-$ S.E.M.). (e) Sesn2 ${ }^{-1-}$ MEFs were transfected with PCMV (empty vector) or pCMV-Flag-Sesn2 for $24 \mathrm{~h}$, and treated with $20 \mathrm{mM}$ 2-DG for the indicated times. The signal of the cleaved form of PARP and caspase 3 were normalized by the signal of erk2 and quantified using MultiGauge software

et al. $^{28}$ demonstrated that the phosphorylation of Akt is not due to the inhibition of glycolysis and is independent of AMPK. Similarly to this study, we show that the treatment with the PI3K inhibitor, LY294002, inhibited 2-DG-induced Akt phosphorylation, suggesting that $\mathrm{PI} 3 \mathrm{~K}$ activity is required for this process. Another possibility is that 2-DG activates Akt via insulin-like growth factor 1; indeed, 2-DG has been shown to dissociate IGF-1 from IGF-binding protein 3 (IGFBP3) so that the free form of IGF-1 could be released from the IGF-1IGFBP3 complex to activate IGF-1 receptor signaling.

Interestingly, the status of PTEN does not seem to interfere with the induction of Sesn2 upon 2-DG treatment as Sesn2 expression is increased in both PTEN-deficient cells (LNCaP, PC3 and DU145) and PTEN-proficient cells (Mefs). We hypothesized that eventhough Akt activity is high in PTENdeficient cells, 2-DG is still able to activate Akt to regulate Sesn2 protein level.
We demonstrate that Sesn2 is required for the downregulation of mTOR by $2-D G$ and metformin. However, Sesn2 does not mediate the effects of HBSS on mTOR. HBSS medium does not contain glucose but also does not contain amino acids. Therefore, we suggest that Sesn2 mediates differentially its effects on mTOR depending on the nature, the 'intensity' of the energetic stress and the metabolic pathway affected. Indeed, we have shown previously that AICAR (5amino-1- $\beta$-D-ribofuranosyl-imidazole-4-carboxamide) and metformin, two energetic stress inducers, trigger different cellular responses in the same cell line. ${ }^{29}$ Besides, depending on the concentration, 2-DG has been shown to trigger autophagy or apoptosis. ${ }^{19,30}$ Here, we work with high concentration $(20 \mathrm{mM})$ of $2-\mathrm{DG}$ that induces apoptosis, in the same cells, we demonstrated previously that $1 \mathrm{mM}$ of 2 DG triggers autophagy. The inhibition of mTOR, mediated by Sesn2, participates to the protective response against 
a
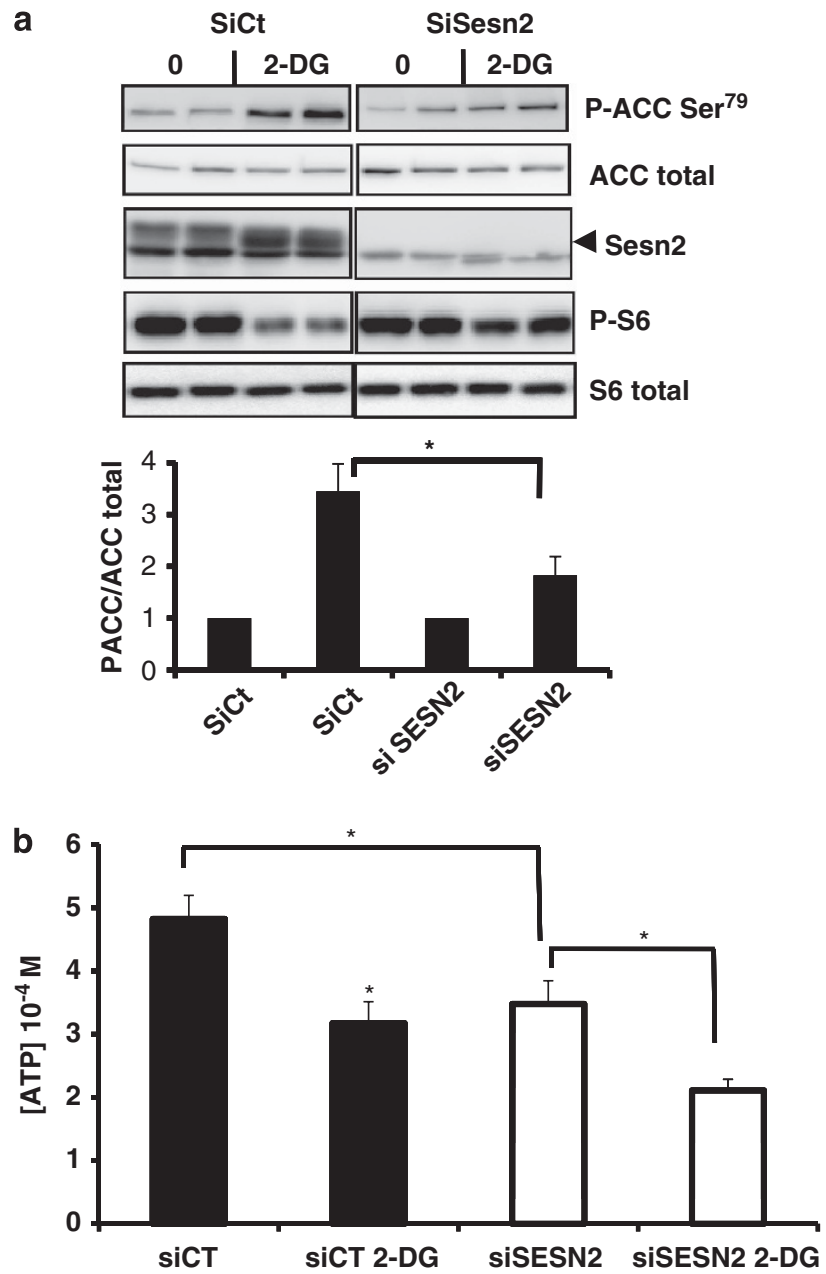

Figure 6 Sesn2 knockdown affects AMPK activity and intracellular ATP levels. (a) LNCaP cells were transfected with siControl (siCt) or siSesn2, and treated for $8 \mathrm{~h}$ with $20 \mathrm{mM}$ 2-DG before immunoblotting. The graph represents the means of three independent experiments with each condition in duplicate. The differences were significant with $P<0.05\left(^{*}\right)$. (b) Intracellular ATP concentration in LNCaP cells transfected with siCt or siSesn2, and treated for $8 \mathrm{~h}$ with $20 \mathrm{mM}$ 2-DG. The results are the mean $+/-$ S.E.M. of five independent experiments. The differences were significant with $P<0.05\left({ }^{*}\right)$

apoptosis. ${ }^{31,32}$ The role of mTOR in apoptosis is controversial, in some models inhibition of mTOR sensitizes to apoptosis, ${ }^{33,34}$ while in other cell lines it reduces apoptosis. ${ }^{30}$ To explain this discrepancy, one can hypothesize that mTOR as a master regulator of protein synthesis may control differentially depending on cell lines and metabolic stress the expression of pro and anti-apoptotic genes. In LNCaP cells, similarly to Sesn2 invalidation, we observed that the downregulation of mTOR with siRNA sensitizes cells to 2-DGinduced apoptosis (data not shown).

It was shown recently that 2-DG stimulates autophagy or apoptosis through ER stress response. ${ }^{21,30}$ We demonstrate that Sesn2 is not implicated in the induction of ER stress by 2 DG (Figure 6a). The mechanism of induction of apoptosis induced by 2-DG is poorly known. Ramirez-Peinado et al. ${ }^{21}$ showed that ER stress rather than ATP depletion induces apoptosis in a Noxa-dependent manner. 3-Bromopyruvate (another inhibitor of glycolysis) triggers apoptosis through the

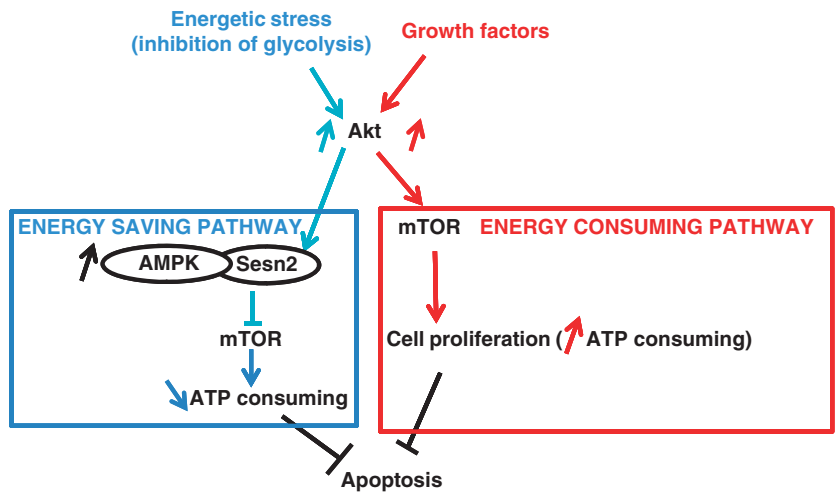

Figure 7 Sesn2, a new sensor of energetic stress. Scheme of our hypothesis, the inhibition of glycolysis induces Sesn2 expression via the anti-apoptotic kinase Akt. The upregulation of Sesn2 is required for the downregulation of the energyconsuming kinase mTOR. Growth factors activate Akt, which upregulate mTOR to promote cell proliferation

dephosphorylation of the pro-apoptotic protein Bad. ${ }^{23}$ In our model, we did not see any significant modification of Noxa and Bad expression in microarray analysis (data not shown).

Akt is known to activate anabolic processes to support cell proliferation. ${ }^{35}$ Intriguingly, we show that Akt by inducing Sesn2 expression participates to the inhibition of a biosynthesis process (mTORC1). In brief, 2-DG activates an energyconsuming pathway (Akt) that triggers an energy saving pathway (inhibition of mTOR via Sesn2). We suggest that a subtle regulation of Akt activity supports this dual action. We hypothesize that Akt by inducing Sesn2 activates a negative feedback loop, which help to refrain its activation effects on mTORC1, thus limiting energy-consuming pathways. This hypothesis is in accordance with a decrease of Akt activity after a $24 \mathrm{~h}$ treatment with 2-DG and a role for Sesn2 in the modulation of anabolism. It was reported recently that FoxO downregulates mTORC1 via Sesn3 and activates Akt to maintain energy homeostasis. ${ }^{18}$ We show here that Sesn3 is not modulated by 2-DG (Figure 3b). We demonstrate that Sesn2 participate to the maintenance of energy homeostasis to protect against cell death; however, whether or not in our model, FoxO is implicated remains to be determined. A better understanding of the mechanism underlying the regulation of Sesn2 by Akt will help to elucidate this issue in future studies.

We identified a new function for Sesn2. Indeed, we obtained evidences in several cell lines that invalidation of Sesn2 sensitizes cells to energetic stress-induced apoptosis, and more importantly, that re-expression of Sesn2 in Sesn2deficient cells protects against cell death. The role of Sesn2 as a pro- or anti-apopotic protein is controversial. Previous studies have shown that Sesn2 expression inhibits cell growth and proliferation in response to a genotoxic stress. ${ }^{15,36}$ However, in the same study, the expression of Sesn2 protected MCF-7 cells against ischemia/low glucose and $\mathrm{H}_{2} \mathrm{O}_{2}$. These results reveal that Sesn2 has a different role depending on the nature of the stress, and our study demonstrates that Sesn2 counteracts the deleterious effects of the inhibition of glycolysis by 2-DG.

We demonstrate that invalidation of Sesn2 aggravates intracellular depletion of ATP in response to 2-DG. This is in 
accordance with the function of Sesn2, which inhibits mTOR and limits anabolic reactions, thus initiating an energy saving process (Figure 7). Interestingly, we show that the knockdown of Sesn2 by itself decreases basal ATP concentration showing that Sesn2 is required for the maintenance of energy homeostasis. Despite this diminution of ATP, we did not observe an increase of ACC phosphorylation. This is in line with the role of Sesn2, which is required for the activation of AMPK. ${ }^{15}$

Targeting glycolysis and cancer cell metabolism is a new promising strategy to fight cancer. ${ }^{37}$ Indeed, several studies associate inhibitors of glycolysis with classical chemotherapeutic agents to improve their efficiency. Although 2-DG alone is poorly effective in vivo, it was successfully used in combination with chemotherapeutic agents, radiotherapy or metformin in vitro and in animal models. ${ }^{19,38,39}$ Consequently, the resistance to energetic stress-induced apoptosis is an important issue in cancer biology. Our study demonstrates that Sesn2 mediates this resistance against 2DG; therefore, a low expression of Sesn2 in tumors should be predictive of a better response to therapies targeting cancer cell metabolism.

\section{Materials and Methods \\ Cell lines and culture conditions. Cells were cultured in RPMI 1640 (LNCaP) or Dulbecco's modified Eagle's medium (Invitrogen, Carlsbad, CA, USA) for other cell lines. Both media contain $4500 \mathrm{mg} / 1$ glucose supplemented with $10 \%$ fetal bovine serum, $100 \mathrm{U} / \mathrm{ml}$ penicillin, $100 \mathrm{mg} / 1$ streptomycin and $2 \mathrm{mM}$ glutamine. Sesn $2^{-1-}$ and sesn2 ${ }^{+1+}$ MEFs were freshly thawed before each experiment.}

Chemicals. 2-DG (Sigma Chemical Co., St Louis, MO, USA) was dissolved in culture media. Akt1/2 inhibitor, bleomycin and staurosporin were purchased from Calbiochem, Merck, Darmstadt, Germany. Doxycyclin (VWR Scientific, Radnor, PA, USA) and Z-VAD fmk (Santa Cruz Biotechnology, Santa Cruz, CA, USA) were dissolved in DMSO, Hank's Balanced Salt Solution (HBSS, Invitrogen).

Cell transfection. Cells were transfected with Lipofectamine RNAi max (Invitrogen), using three different siRNA targeting Sesn2 (Applied Biosystems, Ambion, Carlsbad, CA, USA and Dharmacon, Lafayette, CO, USA). siRNA against p53, Akt1 and Akt2 were from Applied Biosystems, Ambion. LNCaP and MEFs cells were transfected with pCMV-Flag-Sesn2 or with pCMV-empty-vector plasmids using Lipofectamine 2000 or Jet PEI (Polyplus, Illkirch, France).

Measurement of ATP concentration. ATP concentration was measured by luciferase activity using the kit from Roche Applied Science (Meylan, France) and normalized to protein concentration. ${ }^{19}$

Cell analysis. Cell extracts were prepared using lysis buffer. ${ }^{29}$ Immunoblotting was performed with antibodies against Sesn2 (ProteinTech, Chicago, IL), Thr ${ }^{172}$ P-AMPK, P-S6 ribosomal protein, S6 ribosomal protein, AMPK, Caspase 3, ACC, Akt, Ser ${ }^{473}$ P-Akt, Thr ${ }^{308}$ P-Akt, Ser ${ }^{79}$ P-ACC and Thr ${ }^{1462}$ P-TSC2 (Cell Signaling Technology, Danvers, MA, USA); p53, PARP, ERK2 and HSP90 (Santa Cruz Biotechnology); SESN3 (Abcam, Cambridge, UK); and $\alpha$-tubulin (Sigma Chemical Co.).

Caspase 3 assay. Caspase 3 activity was fluorimetrically measured in presence or not of Ac-DEVD-CHO (caspase 3 inhibitor) (Calbiochem, Merck). Enzyme activities were expressed in relative intensity per minute and per milligram of protein. ${ }^{29}$

AnnexinV/PI staining assay. Apoptosis was assessed by measuring membrane redistribution of phosphatidylserine using an annexin V-FITC apoptosis detection kit (Roche Diagnostics, Mannheim, Germany). According to the protocol kit, cells were collected, washed twice with PBS and resuspended in $500 \mu \mathrm{l}$ of staining solution containing FITC-conjugated annexin V antibody and PI.
After incubation on ice for 30 min, cells were analyzed by flow cytometry. Basal apoptosis and necrosis were identically determined on untreated cells.

\section{Conflict of Interest}

The authors declare no conflict of interest.

Acknowledgements. We thank Pierre Roux for the $p 53^{-1-}$ MEFs. We thank Mireille Cormont, Sophie Giorgetti-Peraldi, Béatrice Bailly Maitre, Jean Ehrland Ricci and Yannick Le Marchand Brustel for scientific discussion. This study was supported by The European Foundation for the Study of Diabetes (EFSD) and INCA (grant 2010-219 and 2010-214). IB-S was supported by 'La Ligue Nationale contre le cancer'. IB-S and AP received the 'Prix Jeune Chercheur' of the Bettencourt foundation. BD is supported by INCA grant 2010-219. FB and J-FT are investigators of the Center National de la Recherche Scientifique (CNRS).

1. Warburg O. On the origin of cancer cells. Science 1956; 123: 309-314.

2. Karczmar GS, Arbeit JM, Toy BJ, Speder A, Weiner MW. Selective depletion of tumor ATP by 2-deoxyglucose and insulin, detected by $31 \mathrm{P}$ magnetic resonance spectroscopy. Cancer Res 1992; 52: 71-76.

3. Simons AL, Mattson DM, Dornfeld K, Spitz DR. Glucose deprivation-induced metabolic oxidative stress and cancer therapy. J Cancer Res Ther 2009; 5(Suppl 1): S2-S6.

4. Caro-Maldonado A, Tait SW, Ramirez-Peinado S, Ricci JE, Fabregat I, Green DR et al. Glucose deprivation induces an atypical form of apoptosis mediated by caspase-8 in BaxBak-deficient cells. Cell Death Differ 2010; 17: 1335-1344.

5. Vander Heiden MG, Plas DR, Rathmell JC, Fox CJ, Harris MH, Thompson CB. Growth factors can influence cell growth and survival through effects on glucose metabolism. Mol Cell Biol 2001; 21: 5899-5912.

6. Gonin-Giraud S, Mathieu AL, Diocou S, Tomkowiak M, Delorme G, Marvel J. Decreased glycolytic metabolism contributes to but is not the inducer of apoptosis following IL-3starvation. Cell Death Differ 2002; 9: 1147-1157.

7. Lee YJ, Galoforo SS, Berns CM, Tong WP, Kim HR, Corry PM. Glucose deprivationinduced cytotoxicity in drug resistant human breast carcinoma MCF-7/ADR cells: role of c-myc and bcl-2 in apoptotic cell death. J Cell Sci 1997; 110(Pt 5): 681-686.

8. Alves NL, Derks IA, Berk E, Spijker R, van Lier RA, Eldering E. The Noxa/Mcl-1 axis regulates susceptibility to apoptosis under glucose limitation in dividing T cells. Immunity 2006; 24: 703-716.

9. Yecies JL, Zhang HH, Menon S, Liu S, Yecies D, Lipovsky Al et al. Akt stimulates hepatic SREBP1C and lipogenesis through parallel mTORC1-dependent and independent pathways. Cell Metab 2011; 14: 21-32.

10. Wong KK, Engelman JA, Cantley LC. Targeting the PI3K signaling pathway in cancer. Curr Opin Genet Dev 2010; 20: 87-90.

11. Zha J, Harada H, Yang E, Jockel J, Korsmeyer SJ. Serine phosphorylation of death agonist BAD in response to survival factor results in binding to 14-3-3 not BCL-X(L). Cell 1996; 87: 619-628.

12. Cardone MH, Roy N, Stennicke HR, Salvesen GS, Franke TF, Stanbridge E et al. Regulation of cell death protease caspase-9 by phosphorylation. Science 1998; 282 : 1318-1321.

13. Tang ED, Nunez G, Barr FG, Guan KL. Negative regulation of the forkhead transcription factor FKHR by Akt. J Biol Chem 1999; 274: 16741-16746.

14. Manning BD, Tee AR, Logsdon MN, Blenis J, Cantley LC. Identification of the tuberous sclerosis complex-2 tumor suppressor gene product tuberin as a target of the phosphoinositide 3-kinase/akt pathway. Mol Cell 2002; 10: 151-162.

15. Budanov AV, Karin M. p53 target genes sestrin1 and sestrin2 connect genotoxic stress and mTOR signaling. Cell 2008; 134: 451-460.

16. Zhou H, Li XM, Meinkoth J, Pittman RN. Akt regulates cell survival and apoptosis at a postmitochondrial level. J Cell Biol 2000; 151: 483-494.

17. Zhong D, Liu X, Schafer-Hales K, Marcus Al, Khuri FR, Sun SY et al. 2-Deoxyglucose induces Akt phosphorylation via a mechanism independent of LKB1/AMP-activated protein kinase signaling activation or glycolysis inhibition. Mol Cancer Ther 2008; 7: 809-817.

18. Chen CC, Jeon SM, Bhaskar PT, Nogueira V, Sundararajan D, Tonic I et al. FoxOs inhibit mTORC1 and activate Akt by inducing the expression of Sestrin3 and Rictor. Dev Cell 2010; 18: 592-604.

19. Ben Sahra I, Laurent K, Giuliano S, Larbret F, Ponzio G, Gounon P et al. Targeting cancer cell metabolism: the combination of metformin and 2-deoxyglucose induces p53dependent apoptosis in prostate cancer cells. Cancer Res 2010; 70: 2465-2475.

20. Budanov AV, Sablina AA, Feinstein E, Koonin EV, Chumakov PM. Regeneration of peroxiredoxins by p53-regulated sestrins, homologs of bacterial AhpD. Science 2004; 304: $596-600$.

21. Ramirez-Peinado S, Alcazar-Limones F, Lagares-Tena L, El Mjiyad N, Caro-Maldonado A, Tirado $\mathrm{OM}$ et al. 2-Deoxyglucose induces Noxa-dependent apoptosis in alveolar rhabdomyosarcoma. Cancer Res 2011; 71: 6796-6806.

22. Munoz-Pinedo C, El Mjiyad N, Ricci JE. Cancer metabolism: current perspectives and future directions. Cell Death Dis 2012; 3: e248. 
23. Xu RH, Pelicano H, Zhou Y, Carew JS, Feng L, Bhalla KN et al. Inhibition of glycolysis in cancer cells: a novel strategy to overcome drug resistance associated with mitochondrial respiratory defect and hypoxia. Cancer Res 2005; 65: 613-621.

24. Yao R, Cooper GM. Requirement for phosphatidylinositol-3 kinase in the prevention of apoptosis by nerve growth factor. Science 1995; 267: 2003-2006.

25. Wei $\mathrm{H}$, Vander Heide RS. Heat stress activates AKT via focal adhesion kinase-mediated pathway in neonatal rat ventricular myocytes. Am J Physiol Heart Circ Physiol 2008; 295: H561-H568.

26. Guha M, Fang JK, Monks R, Birnbaum MJ, Avadhani NG. Activation of Akt is essential for the propagation of mitochondrial respiratory stress signaling and activation of the transcriptional coactivator heterogeneous ribonucleoprotein A2. Mol Biol Cell 2010; 21: 3578-3589.

27. Manning BD, Cantley LC. AKT/PKB signaling: navigating downstream. Cell 2007; 129 1261-1274

28. Zhong D, Xiong L, Liu T, Liu X, Liu X, Chen J et al. The glycolytic inhibitor 2-deoxyglucose activates multiple prosurvival pathways through IGF1R. J Biol Chem 2009; 284: 23225-23233.

29. Ben Sahra I, Laurent K, Loubat A, Giorgetti-Peraldi S, Colosetti P, Auberger P et al. The antidiabetic drug metformin exerts an antitumoral effect in vitro and in vivo through a decrease of cyclin D1 level. Oncogene 2008; 27: 3576-3586.

30. Xi H, Kurtoglu M, Liu H, Wangpaichitr M, You M, Liu X et al. 2-Deoxy-D-glucose activates autophagy via endoplasmic reticulum stress rather than ATP depletion. Cancer Chemother Pharmacol 2011; 67: 899-910.
31. Gonzalez-Rodriguez A, Alba J, Zimmerman V, Kozma SC, Valverde AM. S6K1 deficiency protects against apoptosis in hepatocytes. Hepatology 2009; 50: 216-229.

32. Ravikumar B, Berger Z, Vacher C, O'Kane CJ, Rubinsztein DC. Rapamycin pre-treatment protects against apoptosis. Hum Mol Genet 2006; 15: 1209-1216.

33. Gu L, Zhou C, Liu H, Gao J, Li Q, Mu D et al. Rapamycin sensitizes T-ALL cells to dexamethasone-induced apoptosis. J Exp Clin Cancer Res 2010; 29: 150

34. Castedo M, Ferri KF, Kroemer G. Mammalian target of rapamycin (mTOR): pro- and antiapoptotic. Cell Death Differ 2002; 9: 99-100.

35. Yuan TL, Cantley LC. PI3K pathway alterations in cancer: variations on a theme. Oncogene 2008; 27: 5497-5510.

36. Budanov AV, Shoshani T, Faerman A, Zelin E, Kamer I, Kalinski $\mathrm{H}$ et al. Identification of a novel stress-responsive gene Hi95 involved in regulation of cell viability. Oncogene 2002; 21: 6017-6031.

37. Kroemer G, Pouyssegur J. Tumor cell metabolism: cancer's Achilles' heel. Cancer Cell 2008; 13: 472-482.

38. Cheong JH, Park ES, Liang J, Dennison JB, Tsavachidou D, Nguyen-Charles C et al. Dual inhibition of tumor energy pathway by 2-deoxyglucose and metformin is effective against a broad spectrum of preclinical cancer models. Mol Cancer Ther 2011; 10: 2350-2362.

39. Simons AL, Ahmad IM, Mattson DM, Dornfeld KJ, Spitz DR. 2-Deoxy-D-glucose combined with cisplatin enhances cytotoxicity via metabolic oxidative stress in human head and neck cancer cells. Cancer Res 2007; 67: 3364-3370.

Supplementary Information accompanies the paper on Cell Death and Differentiation website (http://www.nature.com/cdd) 